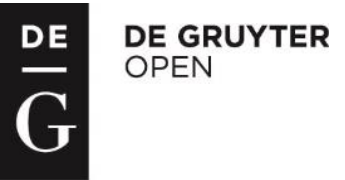

DOI: $10.1515 /$ dch-2015-0004

\title{
Clonal evolution in a patient with aplastic anemia - case report
}

\author{
Melen Brinza $^{1, *}$; Cerasela Jardan ${ }^{1}$; Didona Vasilache ${ }^{1}$; Camelia Dobrea ${ }^{1,2}$; Daniel Coriu ${ }^{1,2}$ \\ 1. Department of Hemathology, Fundeni Clinical Institute, Bucharest, Romania \\ 2. University of Medicine and Pharmacy "Carol Davila”, Bucharest, Romania
}

\begin{abstract}
Background: Aplastic anemia ( $A A)$ is a rare and serious disease characterized by pancytopenia and hypoplastic bone marrow in the absence of infiltrates/fibrosis. It occurs more frequently in childhood and young adulthood (10-30 years) and with older age ( $>60$ years), with equal distribution among men and women. As hypoplastic myelodysplastic syndromes (hMDS) are also associated with cytopenia and hypocellular marrow, they may be difficult to differentiate from AA. The presence of dysplastic features (others than erythroid) and/or blast cells $>5 \%$ is essential to distinguish hMDS from AA. Cytogenetic tests may reveal clonal evolution in hMDS. As the two disorders differ greatly in means of management and prognosis, the correct diagnostic is very important.

Case presentation: We report the case of a 39 years old female diagnosed in 2005 (at age 29) with aplastic anemia. She received treatment with corticosteroids, Cyclosporine, blood transfusions and growth factors with partial response and no transfusion independency. After 8 years of evolution she developed dysplastic features within the megakaryocytic and granulocytic lineages and an increase in the blast population. The bone marrow slowly became hypercellular. The treatment with cyclosporine and growth factors was stopped.
\end{abstract}

Key words: aplastic anemia, hypoplastic myelodysplastic syndrome

\section{*Corresponding author}

MelenBrinza, Department of Hematology, Fundeni Clinical Institute, Sos. Fundeni nr. 258, sector 2, Bucharest, Romania, phone:+40745021923, e-mail: melen.brinza@yahoo.com 


\section{Case report}

We report the case of a 39 years old female, without any previous medical history, who was first admitted to the regional hospital in 2005 for heavy periods, low abdominal pain, intense pallor and important weakness and fatigue. The blood tests revealed severe pancytopenia $(\mathrm{Hb} 6.8 \mathrm{~g} / \mathrm{dl}, \mathrm{WBC}$ $2400 / \mathrm{mmc}$ with ANC $<500 / \mathrm{mmc}$, PLT $19.000 / \mathrm{mmc}$ ). Abdominal ultrasound showed a uterine fibroid and a polycystic ovary. She received 4 units of blood and was later referred to our Hematology Department (in May 2005), with the same symptoms. The physical exam found no palpable lymph nodes, no organomegaly.

The first evaluation showed: Hb $9.2 \mathrm{~g} / \mathrm{dl}$, Ht 28.5\%, MCV $97.8 \mathrm{fl}$, ANC 350/mmc, PLT 10.000/mmc, S12 L80 M8; normal hepatic and renal tests, negative Coombs test, negative Ham and sucrose test, normal ferritin, B12 vitamin and folic acid. Anticardiolipin antibodies - absent.Bone marrow aspirate (Fig.1) and biopsy showed hypocellularity, prominent fat cells, reduced granulocytic and erythrocytic cells, very rare megakaryocytes; frequent mature lymphocytes (38\%) and reactive plasma cells $(20 \%)$; absent fibrosis; CD34+ cells $<5 \%$ (Fig. 1 )

The cytogenetic exam was inconclusive due to insufficient metaphases.

Serum erythropoietin (EPO): $45 \mathrm{mU} / \mathrm{ml}$

In vitro cell cultures showed very reduced cell grow and colony formation (spontaneous and after growth factors).

The chest X-Ray revealed no abnormalities.
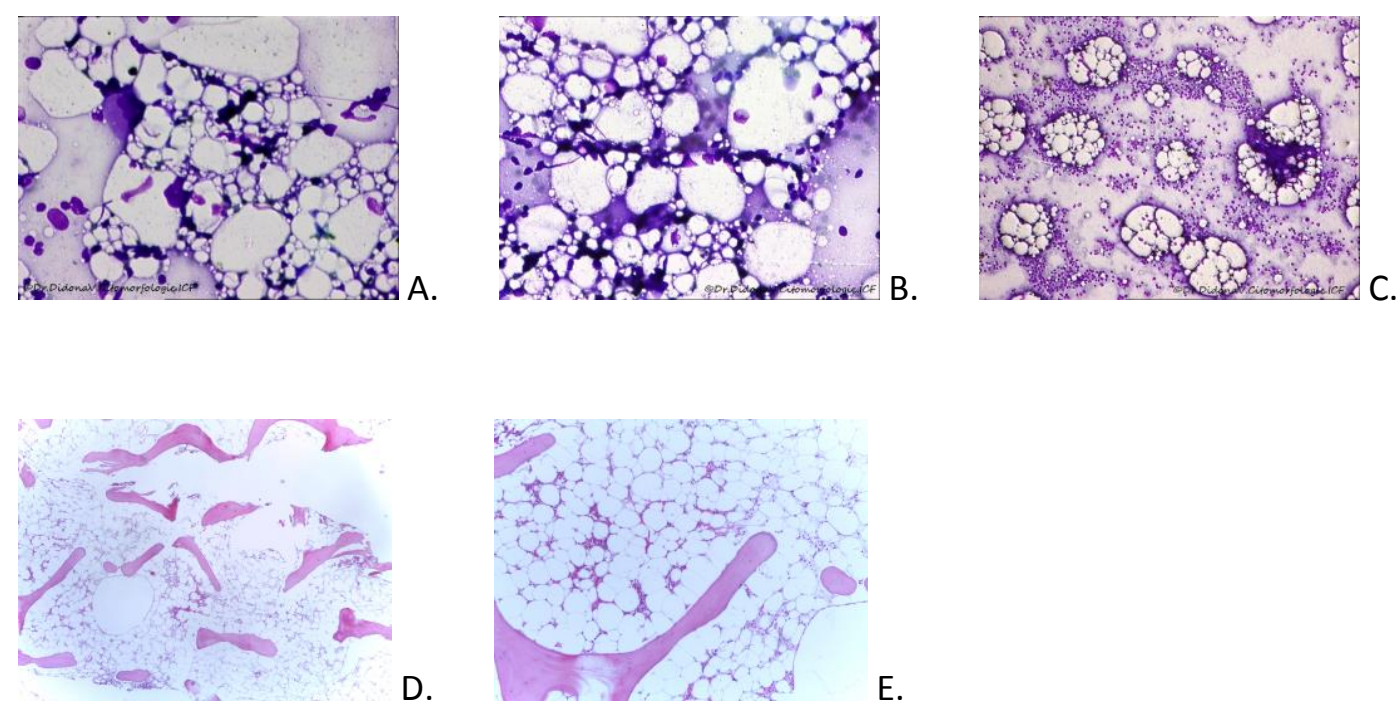

Fig.1 (hypocellular marrow, prominent fat cells, frequent mature lymphocytes; A, B, C- bone marrow aspirate; D, E- bone marrow biopsy)

Based on these test results we established the diagnostic of severe aplastic anemia. 
The treatment recommendations for a patient with this diagnostic are better explained in the figure bellow (Fig 2).

Our patient had no HLA compatible siblings. No anti-thymocyte globulin (ATG) available in our hospital at that time. So she was put on treatment with Prednisone $1 \mathrm{mg} / \mathrm{Kgc}$, Pyridoxine, Duphaston, $\mathrm{r}$ erythropoietin ( $r$-Epo) $10.000 \mathrm{UI} \times 3 /$ week and substitution (red cell, platelets).

There was no improvement in the patient's clinical and biological status, with persistent severe pancytopenia (Hb $4.1 \mathrm{~g} / \mathrm{dl}$, ANC 560, PLT 10.000, gum bleeds, left eye vision impairment). We repeated the bone marrow biopsy with the same morphological findings. She remained on corticotherapy, substitution and rEPO. In 2006 she was put on immunosuppressive treatment (Cyclosporine 300 mg/day) along with Prednisone and blood products.

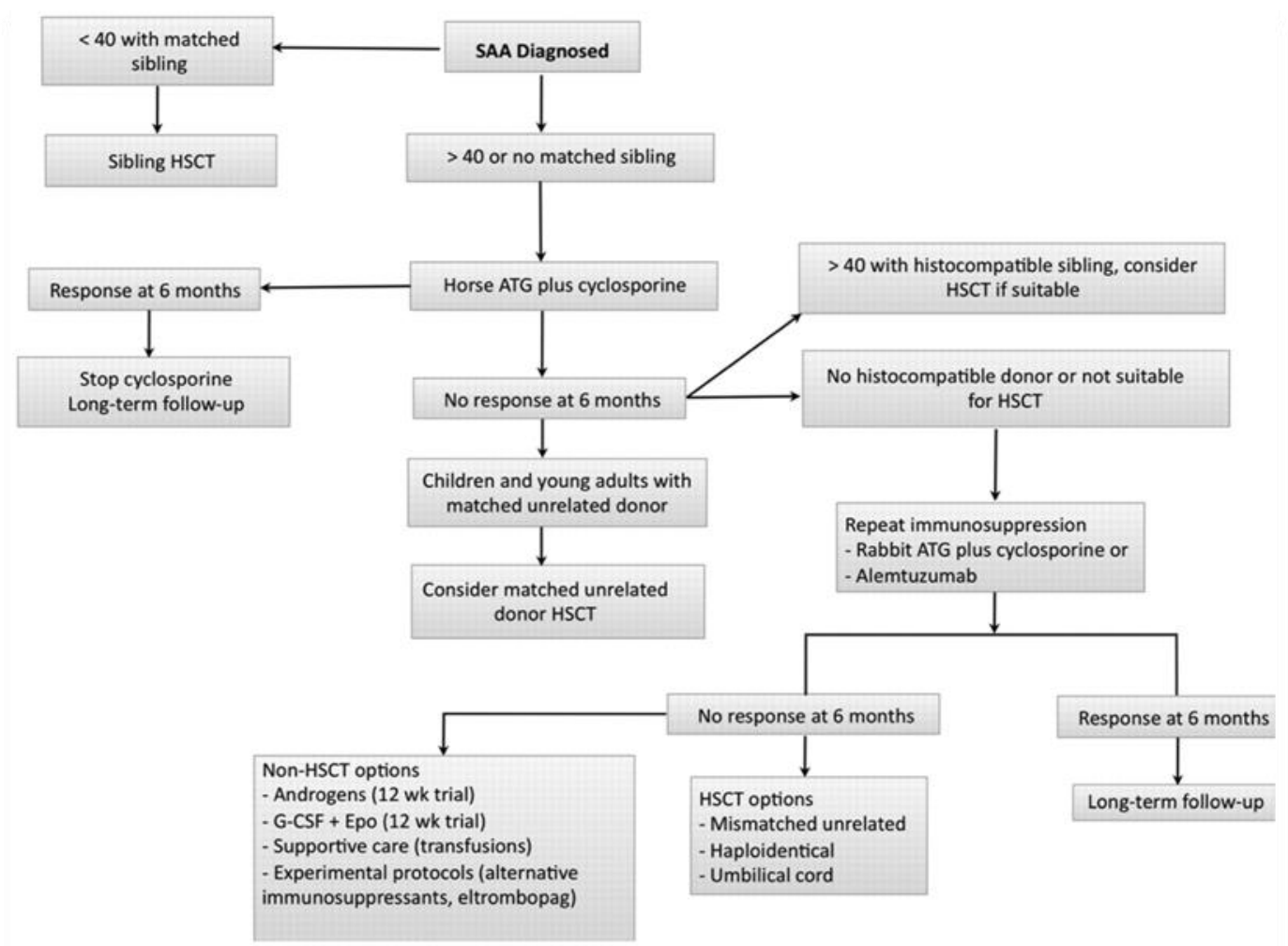

Fig.2 [9]

The patient was lost from our evidence between Apr 2010 and Mar 2013 when she returned with fever, dysphagia, cough, fatigue, Hb $9.4 \mathrm{~g} / \mathrm{dl}$, PLT 69.000/mmc, WBC 2000/mmc, ANC 1460/mmc, cyclosporinemia $275 \mathrm{mcg} / \mathrm{L}$. During these years she received treatment at the regional hospital with Cyclosporine, transfusions (1-2 units blood/month), Dexamethasone occasionally, growth factors (rEPO and G-CSF). The bone marrow biopsy was performed and showed a slight hypocellularity and the presence of megakaryocytes and erythroblasts with dysplastic features, monocytoid cells (Fig.3 and 4). Ham and sucrose tests were negative, ferritin was above $1000 \mathrm{ng} / \mathrm{ml}$. The growth factor administration was stopped. 

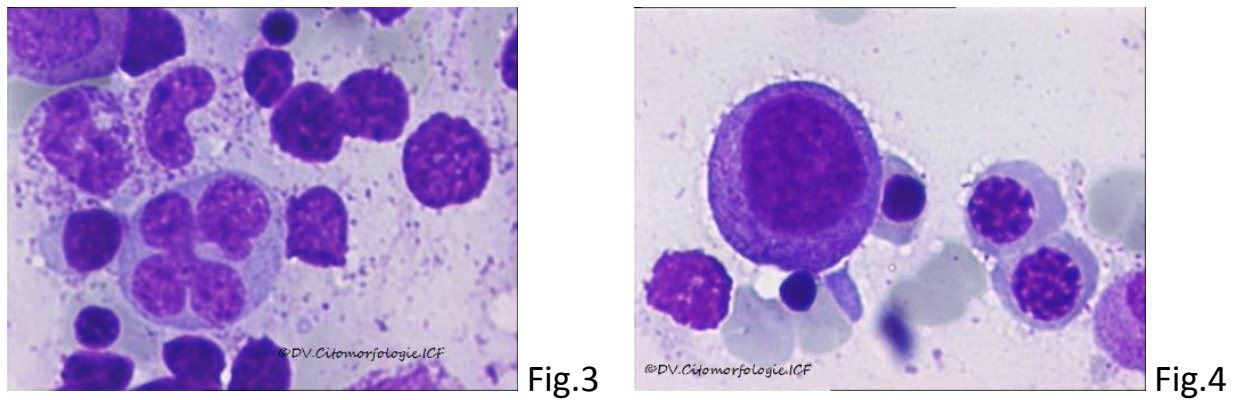

From Nov 2013 the transfusion requirement increased to 2-3 units/month.

In 2014 the patient's clinical and biological status was not improving but getting worse, with $\mathrm{Hb} 6.6$ $\mathrm{g} / \mathrm{dl}$, PLT $47.000 / \mathrm{mmc}$, ANC $500 / \mathrm{mmc}$ and $36 \%$ monocitoid cells in the peripheral blood smears. We repeated the bone marrow biopsy and found hypercellularity, panmyelosis, dysplastic megakaryocytes and erythroblasts (Fig.5, 6 and 7); CD34 + cells 5\%; ferritin $1650 \mathrm{ng} / \mathrm{ml}$.
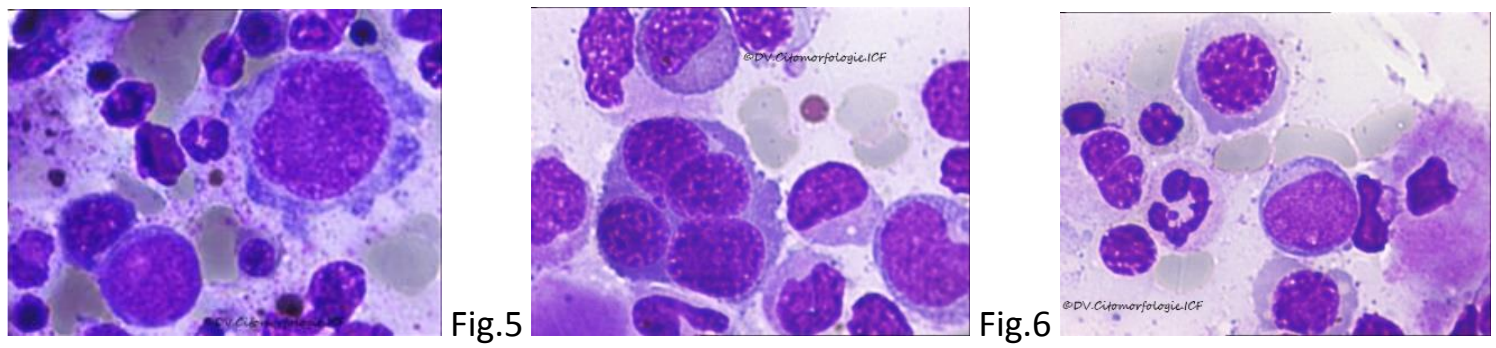

Fig.7

We stopped the therapy with Cyclosporine and started iron chelation with Deferasirox 1000 to 1500 $\mathrm{mg} /$ day. The transfusion need was 3-4 units/month.

The patient's evolution didn't improve, she developed progressive pancytopenia: $\mathrm{Hb} 5.4 \mathrm{~g} / \mathrm{dl}$, PLT $22.000 / \mathrm{mmc}$, WBC $2080 / \mathrm{mmc}$ with $19 \%$ monocytoid cells and $1 \%$ myeloblasts in peripheral blood (in Jan 2015); Hb $4.3 \mathrm{~g} / \mathrm{dl}, \mathrm{WBC} 1150 / \mathrm{mmc}$ and $7 \%$ myeloblasts in PB, PLT 7000/mmc, ANC 350/mmc (Jun 2015).

Bone marrow aspirate: 7-8\% myeloblasts and 4-5\% monocytoid blasts, rare hypogranular granulocytes. Bone marrow biopsy: moderate hypercelullarity, with dysplastic in all lineages (erythroblasts, granulocytes and megakaryocytes). The cytogenetic test was not performed due to technical issues.

We thus diagnosedMyelodysplastic syndrome -RAEB2, following clonal evolution in a patient previously diagnosed with aplastic anemia.

The treatment options at that moment were: 1 . Chemotherapy in low doses (Cytarabine) but with a high risk of infections and with no significant benefits as compared to supportive therapy in terms of AML evolution or overall survival (OS); 2 . Allogeneic stem cell transplant from unrelated donor- the only curative option (30-40\% cases), but associated with great mortality and morbidity; 3 . Hypomethylating agents: Azacytidine $75 \mathrm{mg} / \mathrm{mp} /$ day 7 days every 28 days- may improve hematological status with transfusion independency and postpone the evolution to AML. 4. Supportive therapy: transfusions, iron chelators, antibiotics. Due to the disease's long evolution (8 years), absence of compatible siblings, transfusion history (mostly with non irradiated blood products), the stem cells transplant did not seem a good option for our patient. She continued the supportive treatment, i.e., transfusion therapy and chelator administration, antibiotherapy if needed, with regular monitoring of clinical and biological status 
in our Department. The last visit was in Sep 2015, with a relatively good general status, afebrile, no bleedings, $\mathrm{Hb} 6.2 \mathrm{~g} / \mathrm{dl}$, WBC 3080/mmc, PLT 93.000/mmc.

\section{Discussions:}

The distinctive features of $A A$ are hypocellular bone marrow (with absence of fibrosis or infiltrates) and pancytopenia of various degrees. But these characteristics are also seen in other bone marrow failures: Myelodysplastic Syndrome (MDS) (especially the hypoplastic form) and Paroxistic nocturnal hemoglobinuria (PNH). They can be easily confounded so a clear distinction between them must always be made, especially because they have different evolution and treatment requirement (the risk of transformation in AML is considerably greater in those with MDS).[5], [7] The detection of dysplastic features (other than erythroblastic) at marrow morphology and chromosomal aberration at cytogenetic tests may provide definite proof of hypoplastic MDS (hMDS). (Table 1) [9].

\begin{tabular}{|c|c|c|}
\hline & Aplastic anemia & Hypoplastic MDS \\
\hline Cytopenia & Yes & Yes \\
\hline BM cellularity & Aplastic ( $<10 \%$ cellularity ) or hypocellular & Hypocellular \\
\hline \multicolumn{3}{|l|}{ BM hematopoiesis } \\
\hline Erythropoiesis & Yes in nest, 'hot spots' & Yes \\
\hline Myelopoiesis & Typically decreased & Yes \\
\hline Megakaryopoiesis & Decreased or absent & Yes \\
\hline \multicolumn{3}{|l|}{ Dysplasia } \\
\hline Erythropoiesis & Possible & Possible \\
\hline Myelopoiesis & Normal morphology & Possible \\
\hline Megakaryopoiesis & Normal morphology & Possible \\
\hline Blasts & Absent & Variable \\
\hline CD34+ or CD117+ & Nearly absent & Normal or increased \\
\hline Marrow fibrosis & Absent & Possible \\
\hline Karyotype & Clonal abnormality possible (about $12 \%$ ) & $-7 / \operatorname{del}(7 q)-5 / \operatorname{del}(5 q)$ \\
\hline PNH clone & Frequent & Unusual \\
\hline Splenomegaly at diagnosis & Absent & Possible \\
\hline
\end{tabular}

Table 1: differentiation criteria between AA and hMDS [9] 
However, some consider AA as a clonal hematopoietic disorder and the immunosuppressive therapy may reveal pre-existing abnormalities in those patients who were misclassified initially with AA instead of hMDS. This may have been possible due to the fact that in AA patients were too few cells to be analyzed morphologically and by conventional cytogenetic tests.

Nowadays it is well known that AA is mostly an immune-mediated disorder caused by an abnormal activation of the cytotoxic T cells (that destroy the normal hematopoietic elements); this explains why the main therapy consists of immunosuppressive agents like cyclosporine, antithymocyte globulin (ATG) and steroids, with or without G-CSF (Fig.2). But, at the same time, this treatment may increase the risk of late clonal complications (PNH, MDS, AML transformation) by allowing the abnormal pre-existing clone to escape immunosurveillance and to overgrow and become detectable, allowing AA to be considered as a preleukemic disorder with clonal hematopoiesis and defective stem cells. [6][8]

MDS is characterized by ineffective hematopoiesis, dysplastic features and chromosomal abnormalities. The treatment is more aggressive and includes chemotherapy, hypomethylating agents and allotransplant. Hypocellular MDS is a subgroup that accounts for $7-15 \%$ of MDS cases. The most common chromosomal aberration is monosomy 7. [3] But there are cases of hMDS with normal cytogenetic tests or with good response to immunosuppressive treatment and patients with AA with some cytogenetic abnormalities or poor response to immunotherapy. This makes the distinction between the two (AA and hMDS) even more challenging. Furthermore, the absence of dysplasia does not exclude the possibility of hMDS, especially in patients refractory to the therapy designated for AA or with relatively short interval from the initial diagnosis of AA to the development of MDS/AML.

For the case of our patient, the lack of dysplastic elements in the initial bone marrow aspirate and biopsy was insufficient to exclude the diagnostic of hMDS because.No cytogenetic analysis had beenperformed, so we did not know whether there were any chromosomal abnormalities. The fact that she was refractory to the immunosuppressive therapy (steroids and cyclosporine) is a sign that the patient is more likely to have had hMDS rather than AA at the time of the initial diagnostic. Alternatively, the evolution to MDS was secondary: the therapy with growth factors may have stimulated the defective clone to expand and become detectable after a long period of evolution ( 8 years). The indication of allogeneic stem cell transplant from unrelated donor should have been evaluated earlier in the patient's evolution, but at that time this procedure was not available in our country.

\section{Conclusion:}

There are several reports of patients who first had AA that later transformed into MDS. It is still unclear why this happens, some suggesting the abnormal stimulation by growth factors or the immunosuppression as possible explanations. [1]. Others argue that these patients had hypoplastic MDS at the initial onset of the pancytopenia, but there were not sufficient cells for the dysplasia to be detected on marrow morphology or clonal detection by cytogenetic tests.

Our case underlines the importance of cytogenetic analysis at the time of first presentation of a patient with pancytopenia and hypocellular bone marrow. [2] If the number of metaphases is insufficient, a FISH test for the detection of chromosome 7 abnormalities (the most commonly affected in hMDS) may be useful. Although patients with hMDS may respond to the immunosuppressive therapy, the evolution to $A M L$ is faster and more frequent.

Conflict of interest: Authors state no conflict of interest. 
1. Kaito K, Kobayashi M, Katayama T, et al. Long-term administration of G-CSF for aplastic anaemia is closely related to the early evolution of monosomy $7 \mathrm{MDS}$ in adults. $\mathrm{Br} \mathrm{J}$ Haematol. 1998;103:297-303.

2. Maciejewski JP, Risitano A, Sloand EM, Nunez O, Young NS. Distinct clinical outcomes for cytogenetic abnormalities evolving from aplastic anemia. Blood 2002;99:31293135.

3. Maciejewski JP, Selleri C. Evolution of clonal cytogenetic abnormalities in aplastic anemia. Leuk Lymphoma. 2004; 45:433-440.

4. Appelbaum FR, Barrall J, Storb R, et al. Clonal cytogenetic abnormalities in patients with otherwise typical aplastic anemia. Exp Hematol. 1987; 15:1134-1139

5. Tichelli A, Gratwohl A, Wursch A, Nissen C, Speck B. Late haematological complications in severe aplastic anaemia. Br J Haematol. 1988; 69:413-418.

6. Sergej K, L. Jeffrey M, Patrick A, et al. Therapy may unmask hypoplastic myelodysplastic syndrome that mimics aplastic anemia. Cancer. 2007; 110(7):15201526.

7. John M. Bennett, Attilio o, et al. Diagnostic criteria to distinguish hypocellular acute myeloid leukemia from hypocellular myelodysplastic syndromes and aplastic anemia: recommendations for a standardized approach. Haematologica 2009; 94(2):264-268

8. Seiji K, Akira O, et al. Risk factors for evolution of acquired aplastic anemia into myelodysplastic syndrome and acute myeloid leukemia after immunosuppressive therapy in children. Blood. 2002; 100(3):786-790.

9. Phillip Scheinberg, Neal S. Young, How I treat acquired aplastic anemia. Blood. 2012 Aug 9; 120(6): 1185-1196 